\title{
THE INF-SUP STABILITY OF THE LOWEST ORDER TAYLOR-HOOD PAIR ON AFFINE ANISOTROPIC MESHES
}

\author{
GABRIEL R. BARRENECHEA AND ANDREAS WACHTEL
}

\begin{abstract}
Uniform LBB conditions are of fundamental importance for the finite element solution of problems in incompressible fluid mechanics, such as the Stokes and Navier-Stokes equations. In this work we prove a uniform infsup condition for the lowest order Taylor-Hood pairs $\mathbb{Q}_{2} \times \mathbb{Q}_{1}$ and $\mathbb{P}_{2} \times \mathbb{P}_{1}$ on a family of affine anisotropic meshes. These meshes may contain refined edge and corner patches. We identify necessary hypotheses for edge patches to allow uniform stability and sufficient conditions for corner patches. For the proof, we generalise Verfürth's trick and recent results by some of the authors. Numerical evidence confirms the theoretical results.
\end{abstract}

\section{INTRODUCTION}

The finite element method for the Stokes problem is subject to the satisfaction of the discrete inf-sup condition. For an effective method, the discrete velocity and pressure spaces should be balanced correctly. This balance results in a discrete inf-sup constant that is independent of mesh properties, such as the size and shape of the elements. Concerning the first requirement, there are many finite element pairs which have been proved to be inf-sup stable on regular meshes (see [BBF13] for an extensive review). Concerning the second requirement, for the vast majority of methods the discrete inf-sup constant has only been proved to be independent of the size of the elements, but may depend on the aspect ratio of the elements of the partition.

This work addresses the last point raised in the previous paragraph. The stability of finite element pairs has been less studied in the anisotropic case, but some progress has been made for some specific pairs, especially using discontinuous pressures and, possibly, non-conforming elements for the velocity. For example, in the work [DL08] the authors analyse the Raviart-Thomas element of arbitrary order on triangular and tetrahedral meshes under a maximum angle condition. Another example is [ANS01] where the authors analyse the Crouzeix-Raviart element in anisotropic meshes (see also [AD99]). In [SS98, SSS99] the authors consider quadrilateral and triangular elements for the $h p$-FEM, and analyse pairs like the $\mathbb{Q}_{k+1}^{2} \times \mathbb{Q}_{k-1}(k \geq 1)$ with continuous velocities and discontinuous pressures. Their analysis shows that this family is uniformly inf-sup stable in edge patches, but corner patches were excluded. This is later analytically justified in [AC00], where the quadrilateral element $\mathbb{Q}_{k+1}^{2} \times \mathbb{P}_{k-1}$ was analysed and it was proved that its inf-sup

1991 Mathematics Subject Classification. 65N12, 65N30, 65N50.

Key words and phrases. anisotropic mesh, Taylor, Hood, LBB condition.

This work has been partially supported by the Leverhulme Trust under grant RPG-2012-483. AW gratefully acknowledges the financial support given by the Asociación Mexicana de Cultura A.C.. 
constant depends on the geometric properties of the quadrilaterals in corner patches. In the same reference, the authors propose an enrichment of the velocity space with bubble functions whose degree depends on the aspect ratio. Alternatively, in the recent work [ABW15] a penalisation based on jumps of the pressure was designed to show inf-sup stability independent of the aspect ratio of the partition. For a review and further references on this topic, see [AMR03].

In this work we study the stability of the lowest-order Taylor-Hood pair on a family of affine anisotropic meshes. This pair was originally proposed in [TH73], and was first analysed in [BP79] and [Ver84] for the lowest order on triangles. In the independent works [BF91] and [Ste90] the analysis was extended to higher-order families, both in the triangular and quadrilateral cases, in two space dimensions. Later, in [Bof94, Bof97], the general two- and three-dimensional triangular and tetrahedral cases were addressed. As it is well known (see, e.g., [GR86, Lemma 1.1]), the validity of the inf-sup condition is equivalent to the existence of a Fortin operator. In [Fal08] an explicit construction of this operator is presented for the quadratic and cubic cases. In [MSW13] another explicit construction was built for the lowest order two-dimensional case, and the result was used to justify a preconditioner for a singularly perturbed problem on shape-regular meshes. Some aspects of this explicit construction were later simplified in [Che14].

As far as we are aware, no general proof of stability is available for the TaylorHood pair in the anisotropic case, and only very particular cases have been analysed so far. For instance, the result [BLR12, Lemma 3.1] states an explicit dependency on the aspect ratio, under the assumption that no drastic change of sizes of neighbouring cells in any direction may occur, see [BLR12, Assumption 1]. Moreover, for some anisotropic edge patches, negative results, in the form of numerical experiments, are given in [SSS99] and [AMR03]. In particular, in [AMR03] instabilities are reported for the $\mathbb{P}_{2}^{2} \times \mathbb{P}_{1}$ pair when the aspect ratio tends to zero in certain configurations. These numerical results (and some results presented in the present work) show that a proof of the uniform stability of the Taylor-Hood pair on general anisotropic meshes cannot be given.

Based on the above discussions, the purpose of this paper is to present a proof for a family of affine anisotropic meshes containing patches of parallelograms, and triangular meshes appearing as a result of dividing each parallelogram into two triangles. The results presented below are valid for edge and corner patches (thus improving upon the results from [BLR12]), but they do not cover the case in which the mesh does not have a structure. In particular, our results do not include the case of general quadrilaterals, or when the elements can not be arranged into corner or edge patches. Our main results state that if the partitions have enough internal degrees of freedom, and these are at the right locations, then this element is uniformly inf-sup stable. That is, the inf-sup constant is independent of the aspect ratio and drastic changes of sizes. Finally, we will show by means of numerical experiments that our assumptions are optimal, which complements the results from [AMR03].

The rest of the paper is organised as follows. In $\S 2$ we present the problem of interest and give some notation. Our main results are then stated in $\S 3$ and tested numerically in $\S 4$. The proofs for the quadrilateral case are then presented in $\S 5$, and for the triangular case in $\S 6$. 


\section{PRELIMINARIES AND NOTATION}

Let $\Omega \subset \mathbb{R}^{2}$ be an open, bounded, connected and polygonal domain. Throughout, we use standard notation for Sobolev spaces (see [GR86]), namely, for $D \subset \Omega$, $L^{2}(D)$ (resp., $\left.L_{0}^{2}(D)\right)$ stands for the space of (generalised) functions which are square integrable in $D$ (resp., which belong to $L^{2}(D)$ and have zero mean value in $D), H^{1}(D)\left(H_{0}^{1}(D)\right)$ are elements of $L^{2}(D)$ whose first order derivatives belong to $L^{2}(D)$ (and whose trace is zero on $\partial D$ ). Vector-valued spaces and functions will be denoted using bold-faced letters. The inner product in $L^{2}(D)$ (or $\boldsymbol{L}^{2}(D)$ ) is denoted by $(\cdot, \cdot)_{D}$, with associated norm $\|\cdot\|_{0, D}$, the norm (seminorm) in $H^{1}(D)$ is denoted by $\|\cdot\|_{1, D}\left(|\cdot|_{1, D}\right)$. By virtue of the Poincaré inequality $|\cdot|_{1, D}$ is a norm on $H_{0}^{1}(D)$. Finally, we denote by $\chi_{D}$ the characteristic function of $D$.

A classical result (see [GR86]) is the following inf-sup condition: There exists $\beta_{\Omega}>0$, depending only on $\Omega$, such that

$$
\inf _{q \in M} \sup _{\boldsymbol{v} \in \boldsymbol{V}} \frac{(\operatorname{div} \boldsymbol{v}, q)_{\Omega}}{|\boldsymbol{v}|_{1, \Omega}\|q\|_{0, \Omega}} \geq \beta_{\Omega}>0,
$$

where $\boldsymbol{V} \times M:=\boldsymbol{H}_{0}^{1}(\Omega) \times L_{0}^{2}(\Omega)$. The purpose of this work is to prove the discrete analogue of this result for the lowest order Taylor-Hood element on a family of anisotropic meshes defined in the next section.

2.1. Restrictions on partitions. We require a partition $\mathcal{P}$ of $\Omega$ to have the following properties. We suppose $\Xi$ is a conforming shape regular partition of $\Omega$ into parallelograms (macro elements). These macro element cells are denoted by $\omega$. The partition $\mathcal{P}$ is a conforming refinement of $\Xi$ and may contain edge patches as in Figs. 1(d-f) and corner patches as in Figs. 2(c-d) (for rectangular patches), or affine images of these, as depicted in Fig. 4. Our proof is rigorous for such meshes and the Taylor-Hood pair on their triangulated versions, that is, each parallelogram is divided into two triangles of equal area, see $\S 6$.

Concerning further refined edge patches, in Remark 3.1 we conjecture that a similar proof is valid. We refer to Remark 3.1 and the numerical evidence for more details.

2.2. Finite element spaces. We now define the discontinuous space

$$
\mathbb{Q}_{-\ell, \mathcal{P}}:=\left\{q \in L^{2}(\Omega):\left.q\right|_{K} \in \mathbb{Q}_{\ell}(K) \text { for } K \in \mathcal{P}\right\} \quad \text { for } \quad \ell=1,2,
$$

and for $\omega \subseteq \Omega$ the (locally) continuous spaces

$$
\mathbb{Q}_{\ell, \mathcal{P}}(\omega):=\left\{q \in \mathbb{Q}_{-\ell, \mathcal{P}}: \operatorname{supp} q \subseteq \bar{\omega}\right\} \cap C^{0}(\omega) .
$$

Let $M_{\mathcal{P}}(\omega):=\mathbb{Q}_{1, \mathcal{P}}(\omega) \cap L_{0}^{2}(\omega)$; in the case of $\omega=\Omega$ we write shortly $M_{\mathcal{P}}$. Let $\boldsymbol{V}_{\mathcal{P}}(\omega):=\left[\mathbb{Q}_{2, \mathcal{P}}(\omega)\right]^{2} \cap \boldsymbol{H}_{0}^{1}(\omega)$; in the case of $\omega=\Omega$ we write shortly $\boldsymbol{V}_{\mathcal{P}}$.

Throughout this manuscript, $C$ (with or without subscript) will denote a positive constant, which will be independent of the size, and aspect ratio of the elements of a given partition. The value of such a constant needs not to be equal whenever written in two different places.

\section{MAIN RESULTS}

In this section we state the main results of this work. We restrict the presentation to results on anisotropically partitioned macro-elements $\omega \in \Xi$. Since, given local 
inf-sup conditions and the shape-regularity of $\Xi$, a macro-element technique ensures uniform stability on the whole partition $\mathcal{P}$.

To ease the readability, the proofs of Theorems 3.1 and 3.2, dealing with rectangular edge and corner patches, respectively, are postponed to $\S 5$. The proof of the extension of these results to the parallelogram case is given in Theorem 3.3. Finally, we sketch in $\S 6$ how the proofs can be extended to triangulated edge and corner patches. Before stating the results, we state the main hypothesis for edge patches.

Hypothesis 1. We will suppose that every edge patch contains at least one division in the direction orthogonal to the "long and thin" elements. More precisely, every "long and thin" element is divided into two "shorter" elements.

(a)

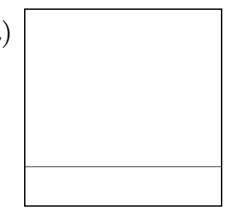

(d)

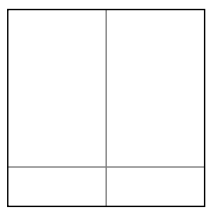

(b)

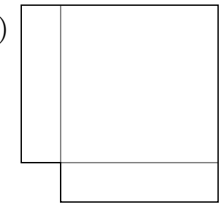

(e)

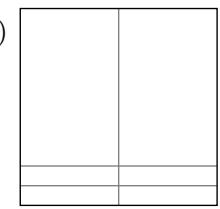

(c)

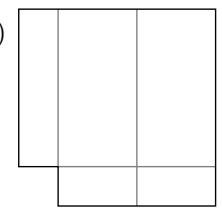

(f)

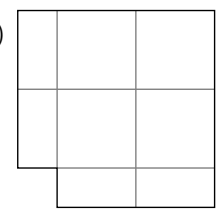

FIG. 1. Edge patches. The cases in the top row do not satisfy Hypothesis 1, but the cases in the bottom row do satisfy it. The examples depicted in (b), (c) and (f) are overlapped edge patches.

Theorem 3.1. Let $\omega \subset \Omega$ be partitioned as an edge patch as depicted in Figs. 1 (d,e,f). Then, there exists a constant $\beta>0$, independent of the shape and size of the elements in $\mathcal{P}$, such that,

$$
\inf _{q \in M_{\mathcal{P}}(\omega)} \sup _{\boldsymbol{v} \in \boldsymbol{V}_{\mathcal{P}}(\omega)} \frac{(\operatorname{div} \boldsymbol{v}, q)_{\omega}}{|\boldsymbol{v}|_{1, \omega}\|q\|_{0, \omega}} \geq \beta .
$$

Remark 3.1. One clear consequence of the proof of Theorem 3.1 is that the infsup constant $\beta$ is the same one for the cases depicted in Figs. 1(d,e). This opens the door to study the case in which an edge patch has been refined further. More precisely, if we say that the edge patch in Fig. 1(d) is refined $r=0$ times, and the patch in Fig. 1(e) is refined $r=1$ times, then, continuing in the same way, i.e., bisecting the long and thin elements $r$ times, we can define a patch that has been refined $r \geq 2$ times, e.g., Fig. 5. Our conjecture, which is supported by numerical evidence shown in Table 4, is that the inf-sup constant (on edge patches) is not affected by the value of $r$, but a formal proof of this fact is lacking.

The next result concerns corner patches. A corner patch will be decomposed as $\omega=\omega_{c} \cup \omega_{E}$ where $\omega_{c}$ is the shape-regular small region (shaded in Fig. 2 for each corner patch) and $\omega_{E}$ is an "overlapped" edge patch as discussed in Theorem 3.1 (see Fig. 1). For the proof of Theorem 3.2, we will need the following assumption. 
Hypothesis 2. The Partition on $\omega_{\boldsymbol{c}} \subset \omega$ is such that the pair $\boldsymbol{V}_{\mathcal{P}}\left(\omega_{\boldsymbol{c}}\right) \times M_{\mathcal{P}}\left(\omega_{\boldsymbol{c}}\right)$ is (uniformly) inf-sup stable with a constant $\beta_{\boldsymbol{c}}$.

(a)

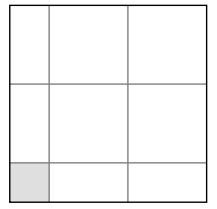

(c)

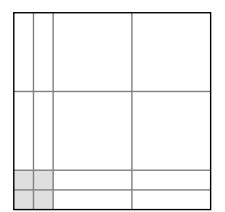

(b)

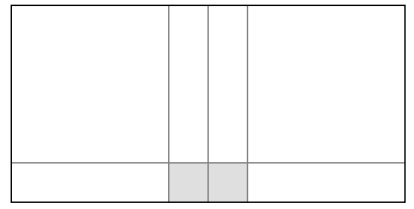

(d)

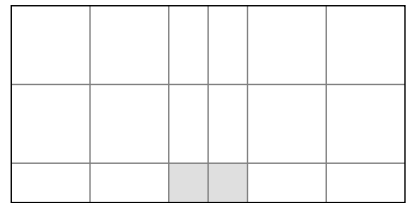

Fig. 2. Corner patches. Case (a) does not satisfy Hypothesis 2, the others do. However, the assumptions of Theorem 3.2 also exclude case (b) since the "overlapped" edge patch does not satisfy Hypothesis 1.

Theorem 3.2. Let $\omega \subset \Omega$ be partitioned into a corner patch, that is, $\omega=\omega_{\boldsymbol{c}} \cup \omega_{E}$ with $\omega_{c}$ and $\omega_{E}$ satisfying Hypothesis 2 and Theorem 3.1, respectively (e.g. as shown in Figs. $2(c-d)$ ). Then, there exists $\beta_{\mathcal{P}}>0$, depending only on $\beta$ (from (2)) and $\beta_{\boldsymbol{c}}$ (from Hypothesis 2), such that

$$
\inf _{q \in M_{\mathcal{P}}(\omega)} \sup _{\boldsymbol{v} \in \boldsymbol{V}_{\mathcal{P}}(\omega)} \frac{(\operatorname{div} \boldsymbol{v}, q)_{\omega}}{|\boldsymbol{v}|_{1, \omega}\|q\|_{0, \omega}} \geq \beta_{\mathcal{P}} .
$$

Remark 3.2. A comment on Hypothesis 2 is in place. In the case of a single corner patch, Hypothesis 2 requires $\omega_{c}$ to be refined (as in Fig. 2(c)). Now, if a corner patch is formed "joining" several patches of the type shown in Fig. 2(a), see for instance Fig. 2(d), then the presence of enough degrees of freedom inside of $\omega_{c}$ ensures that Hypothesis 2 is satisfied without the need of further refinement.

The results presented in Theorems 3.1 and 3.2 remain valid on affine images of allowed edge and corner patches, and meshes that contain such, see for instance, the examples in Fig. 4. The next result shows such an extension.

Theorem 3.3. Let $\hat{\omega}$ be a rectangular edge or corner patch that satisfies the hypotheses of Theorem 3.1 or 3.2, and let $\omega \in \Xi$ be the image of $\hat{\omega}$ under a bijective affine map $\mathcal{F}_{\omega}: \hat{\omega} \rightarrow \omega$. Then, there exists a constant $\beta_{\mathcal{A}}$, depending only on the shape of $\omega$, such that

$$
\inf _{q \in M_{\mathcal{P}}(\omega)} \sup _{\boldsymbol{v} \in \boldsymbol{V}_{\mathcal{P}}(\omega)} \frac{(\operatorname{div} \boldsymbol{v}, q)_{\omega}}{|\boldsymbol{v}|_{1, \omega}\|q\|_{0, \omega}} \geq \beta_{\mathcal{A}} .
$$

Proof. Let $\mathcal{F}_{\omega}(\hat{\boldsymbol{x}})=\mathbb{A} \hat{\boldsymbol{x}}+\boldsymbol{b}$, and let us denote $J:=\operatorname{det}(\mathbb{A}) \neq 0$. Let, for $\hat{\boldsymbol{v}} \in H^{1}(\hat{\omega})^{2}$, $\boldsymbol{v} \in H^{1}(\omega)^{2}$ be its Piola transorm given by

$$
\boldsymbol{v}(\boldsymbol{x}):=|J|^{-1} \mathbb{A} \hat{\boldsymbol{v}}(\hat{\boldsymbol{x}}) \quad \text { where } \quad \boldsymbol{x}=\mathcal{F}_{\omega}(\hat{\boldsymbol{x}}) .
$$


It is well-known (see, e.g., [Dur08, Lemma 3.3]) that

$$
(\operatorname{div} \boldsymbol{v}, q)_{\omega}=(\widehat{\operatorname{div}} \hat{\boldsymbol{v}}, \hat{q})_{\hat{\omega}},
$$

for all $\boldsymbol{v} \in H^{1}(\omega)^{2}$ and all $q \in H^{1}(\omega)$. Here, we have denoted $\hat{q}(\hat{\boldsymbol{x}}):=q\left(\mathcal{F}_{\omega}(\hat{\boldsymbol{x}})\right)=$ $q(\boldsymbol{x})$ and by $\widehat{\mathrm{div}}$ the divergence with respect to the variable $\hat{\boldsymbol{x}}$. This notation will be extended to any derivatives later on.

Next, the chain rule gives

$$
\nabla \boldsymbol{v}(\boldsymbol{x})=|J|^{-1} \mathbb{A} \nabla\left(\hat{\boldsymbol{v}} \circ \mathcal{F}_{\omega}^{-1}\right)(\boldsymbol{x})=|J|^{-1} \mathbb{A} \hat{\nabla} \hat{\boldsymbol{v}}(\hat{\boldsymbol{x}}) \mathbb{A}^{-1},
$$

and then, using the shape-regularity of $\omega$ and $\hat{\omega}$ (to bound the norms of $\mathbb{A}$ and $\mathbb{A}^{-1}$ ) and changing variables we obtain

$$
\begin{aligned}
|\boldsymbol{v}|_{1, \omega}^{2} & =\int_{\omega}|\nabla \boldsymbol{v}(\boldsymbol{x})|^{2} \mathrm{~d} \boldsymbol{x} \\
& =\int_{\omega}|J|^{-2}\left|\mathbb{A} \hat{\nabla} \hat{\boldsymbol{v}}(\hat{\boldsymbol{x}}) \mathbb{A}^{-1}\right|^{2} \mathrm{~d} \boldsymbol{x} \\
& \leq C|J|^{-2} \int_{\omega}\|\mathbb{A}\|_{2}^{2}|\widehat{\nabla} \hat{\boldsymbol{v}}(\hat{\boldsymbol{x}})|^{2}\left\|\mathbb{A}^{-1}\right\|_{2}^{2} \mathrm{~d} \boldsymbol{x} \\
& \leq C_{0}|J|^{-2} \int_{\omega}|\hat{\nabla} \hat{\boldsymbol{v}}(\hat{\boldsymbol{x}})|^{2} \mathrm{~d} \boldsymbol{x} \\
& =C_{0}|J|^{-1}|\hat{\boldsymbol{v}}|_{1, \hat{\omega}}^{2},
\end{aligned}
$$

where the constant $C_{0}$ depends only on the shape of $\omega$. In addition, a change of variables gives

$$
\|q\|_{0, \omega}=|J|^{\frac{1}{2}}\|\hat{q}\|_{0, \hat{\omega}} \cdot
$$

Finally, from Theorems 3.1 and 3.2 (depending whether $\hat{\omega}$ is decomposed into an edge or a corner patch, respectively), there exists a constant $\hat{\beta}>0$ such that, for all $\hat{q} \in M_{\mathcal{P}}(\hat{\omega})$ the following holds

$$
\hat{\beta}\|\hat{q}\|_{0, \tilde{\omega}} \leq \sup _{\hat{\boldsymbol{v}} \in \boldsymbol{V}_{\mathcal{P}}(\hat{\omega})} \frac{(\widehat{\operatorname{div}} \hat{\boldsymbol{v}}, \hat{q})_{\hat{\omega}}}{|\hat{\boldsymbol{v}}|_{1, \hat{\omega}}} .
$$

Hence, using (6), (7), (4), and (5) we obtain, for all $q \in M_{\mathcal{P}}(\omega)$

$$
\begin{aligned}
\hat{\beta}\|q\|_{0, \omega} & =\hat{\beta}|J|^{\frac{1}{2}}\|\hat{q}\|_{0, \hat{\omega}} \\
& \leq|J|^{\frac{1}{2}} \sup _{\hat{\boldsymbol{v}} \in \boldsymbol{V}_{\mathcal{P}}(\hat{\omega})} \frac{(\widehat{\operatorname{div}} \hat{\boldsymbol{v}}, \hat{q})_{\hat{\omega}}}{|\hat{\boldsymbol{v}}|_{1, \hat{\omega}}} \\
& =|J|^{\frac{1}{2}} \sup _{\boldsymbol{v} \in \boldsymbol{V}_{\mathcal{P}}(\omega)} \frac{(\operatorname{div} \boldsymbol{v}, q)_{\omega}}{|\hat{\boldsymbol{v}}|_{1, \hat{\omega}}} \\
& \leq|J|^{\frac{1}{2}} \sup _{\boldsymbol{v} \in \boldsymbol{V}_{\mathcal{P}}(\omega)} \frac{(\operatorname{div} \boldsymbol{v}, q)_{\omega}}{C_{0}^{-1}|J|^{\frac{1}{2}}|\boldsymbol{v}|_{1, \omega}}
\end{aligned}
$$

and the result follows with $\beta_{\mathcal{A}}:=\hat{\beta} C_{0}^{-1}$. 


\section{NumERICAL CONFIRMATION}

In this section we report the discrete inf-sup constants for the Taylor-Hood pairs in different configurations of edge and corner patches. Our aim is to confirm the validity of the results from last section. Then, we introduce the parameter $h$ in such a way that the short side of an anisotropic element is of size $h$, and the long one is $O(1)$ (see Fig. 3 for details). For the refinement in the direction orthogonal to the long and thin element required by Hypothesis 1, we have introduced an extra edge at the midpoint of the long and thin edges (for example, in Fig. 1(e) the extra vertical edge is located at $x=1 / 2$, and in Fig. $1(\mathrm{f})$ at $x, y=(1+h) / 2)$.
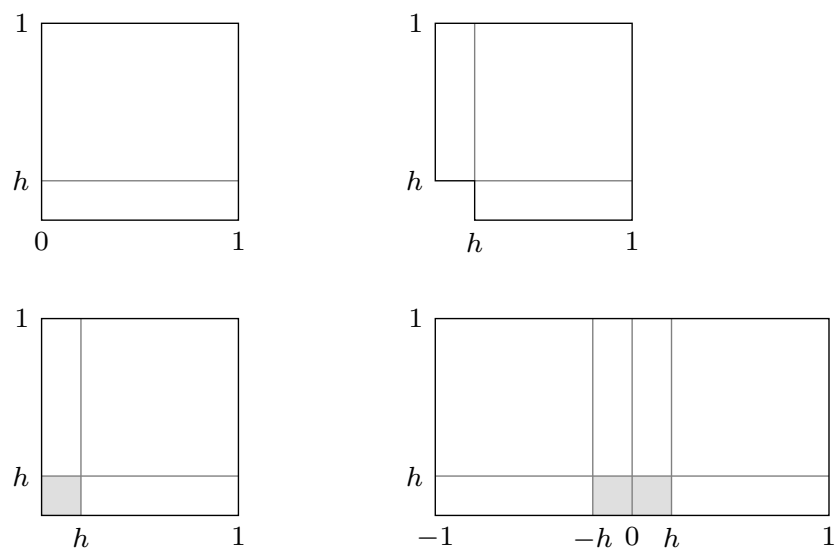

FIG. 3. Parametrised geometries for the coarse partitions of the edge and corner patches shown in Figs. 1 and 2. That is, before subdividing cells so that Hypotheses 1 and 2 are satisfied.

4.1. Results on edge patches. Our first experiments (in Table 1) aim at showing that Hypothesis 1 imposes sufficient (and necessary) requirements on edge patches such that the pair $\mathbb{Q}_{2}^{2} \times \mathbb{Q}_{1}$ is uniformly stable on them. The results reported in the last two columns of Table 1 show that, once the requirements from Hypothesis 1 are fulfilled, the inf-sup constant remains bounded below by a constant independent of $h$. Now, to assess the necessity of this restriction we also report in the first two columns of Table 1 the results for partitions that do not satisfy this hypothesis, where we see that the inf-sup constants degenerate with $h$.

TABle 1. Discrete inf-sup constants of the pair $\mathbb{Q}_{2}^{2} \times \mathbb{Q}_{1}$ on edge patches shown in Fig. 1

\begin{tabular}{ccccc}
\hline$h$ & Fig. 1(a) & Fig. 1(c) & Fig. 1(d) & Fig. 1(f) \\
\hline $10^{-1}$ & $7.12 \cdot 10^{-2}$ & $1.07 \cdot 10^{-1}$ & 0.426 & 0.487 \\
$10^{-2}$ & $7.83 \cdot 10^{-3}$ & $1.17 \cdot 10^{-2}$ & 0.256 & 0.477 \\
$10^{-3}$ & $7.90 \cdot 10^{-4}$ & $1.20 \cdot 10^{-3}$ & 0.208 & 0.469 \\
$10^{-4}$ & $7.90 \cdot 10^{-5}$ & $1.21 \cdot 10^{-4}$ & 0.202 & 0.468 \\
$10^{-5}$ & $7.91 \cdot 10^{-6}$ & $1.21 \cdot 10^{-5}$ & 0.201 & 0.468 \\
\hline
\end{tabular}


4.2. Results on corner patches. In Table 2 we report the results obtained for different configurations of corner patches. We confirm the results of Theorem 3.2 in the sense that whenever the hypotheses imposed on the partitions are satisfied, the inf-sup constant remains bounded below by a constant independent of $h$ (as it can be seen in the last two columns). On the contrary, the first column of Table 2 shows that if the hypotheses are violated, then the inf-sup constant decays with $h$.

TABLE 2. Discrete inf-sup constants of the pair $\mathbb{Q}_{2}^{2} \times \mathbb{Q}_{1}$ on corner patches shown in Fig. 2

\begin{tabular}{cccc}
\hline$h$ & Fig. 2(b) & Fig. 2(c) & Fig. 2(d) \\
\hline $10^{-1}$ & $4.01 \cdot 10^{-1}$ & 0.455 & 0.341 \\
$10^{-2}$ & $1.82 \cdot 10^{-1}$ & 0.406 & 0.324 \\
$10^{-3}$ & $6.07 \cdot 10^{-2}$ & 0.384 & 0.323 \\
$10^{-4}$ & $1.93 \cdot 10^{-2}$ & 0.382 & 0.322 \\
$10^{-5}$ & $6.11 \cdot 10^{-3}$ & 0.381 & 0.322 \\
\hline
\end{tabular}

4.3. The stability on affine meshes. The previous examples were restricted to one single edge, or corner, patch. Next, we show two examples in which edge and corner patches are combined. For this, Fig. 4 shows an affine L-shaped mesh (left) and a snowflake mesh (right). To obtain the left mesh, we took an L-shaped mesh on $[-2,1] \times[0,1] \cup[0,1] \times[-1,0]$ and applied the map $F(\boldsymbol{x}):=\left(\begin{array}{cc}1 & 0 \\ 0.25 & 1\end{array}\right) \boldsymbol{x}$ to all nodes in $[-2,0] \times[0,1]$ and the map $F(\boldsymbol{x}):=\left(\begin{array}{cc}1 & 0.5 \\ 0 & i\end{array}\right) \boldsymbol{x}$ to all nodes in $[0,1] \times[-1,0]$. The snowflake mesh is the union of 5 corner patches with a minimal angle of $72^{\circ}$, its re-entrant corners have a distance of one from the origin. Both meshes have in common that the small edges connected to the origin are of length $h$. Table 3 shows that, once again, the inf-sup constants stay bounded below by a constant independent of the value $h$, as predicted by Theorem 3.3.
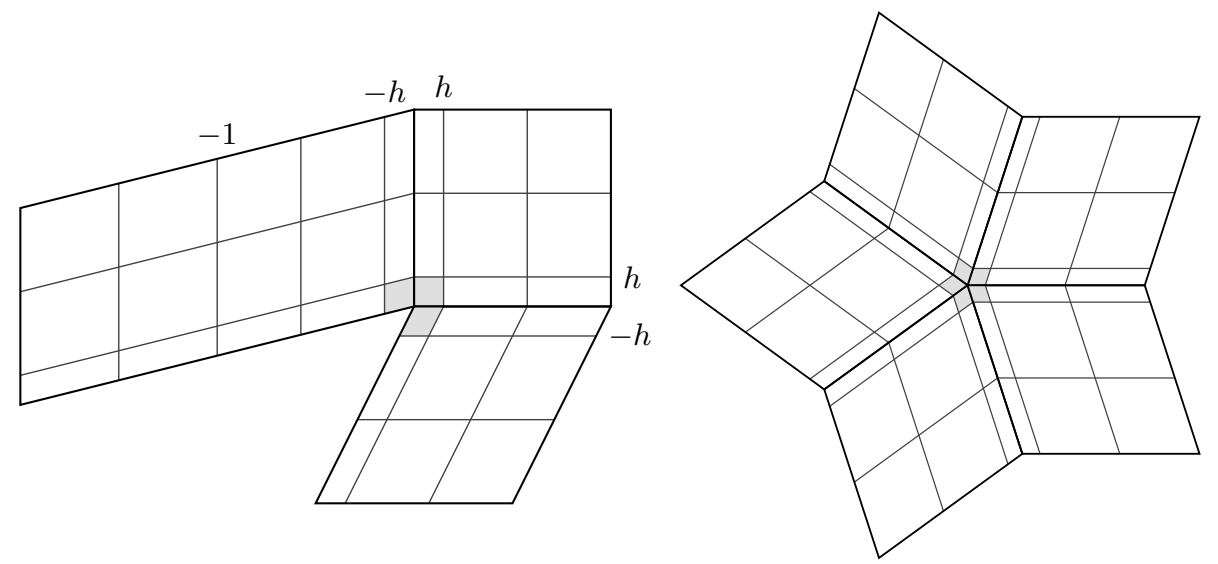

FIG. 4. Meshes with affine corner and edge patches. 
TABLE 3 . Inf-sup constants $\beta_{\mathcal{P}}$ of the $\mathbb{Q}_{2}^{2} \times \mathbb{Q}_{1}$ pair on affine meshes

\begin{tabular}{ccc}
\hline$h$ & Fig. 4(left) & Fig.4(right) \\
\hline $10^{-1}$ & 0.21474 & 0.39513 \\
$10^{-2}$ & 0.21242 & 0.39630 \\
$10^{-3}$ & 0.21135 & 0.39632 \\
$10^{-4}$ & 0.21119 & 0.39632 \\
$10^{-5}$ & 0.21118 & 0.39632 \\
$10^{-6}$ & 0.21113 & 0.39632 \\
\hline
\end{tabular}

4.4. Refined patches. In this section we verify numerically the claims made in Remark 3.1. For this, we consider the refined edge and corner patches depicted in Fig. 5. In Table 4 we report the values of the inf-sup constants. We observe that, as was conjectured in Remark 3.1, for the case of a refined edge patch the inf-sup constants reported in the first two columns remain independent of the number of refinements, thus confirming that conjecture, at least numerically. Now, for the case of a refined corner patch, we see that, as mentioned in Remark 5.4 (on page 17), the value of the inf-sup constants (reported in the third column of Table 4) decreases with the number of refinements, thus confirming the sharpness of the proof of Theorem 3.2.
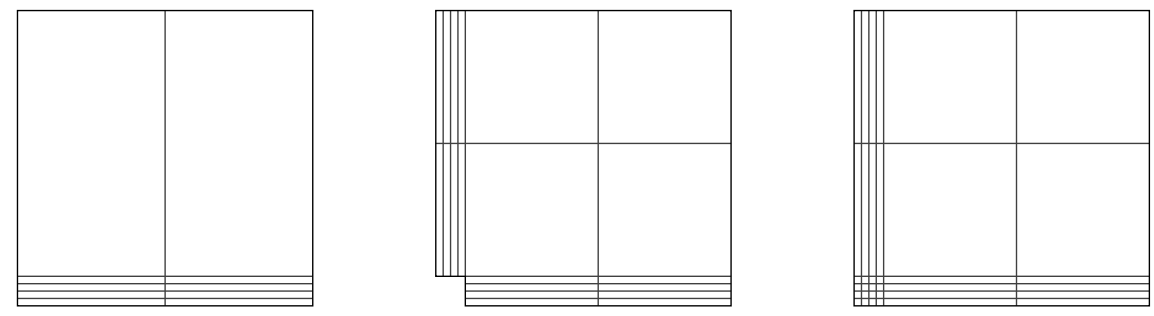

Fig. 5. These patches are refinements of level $r=2$ of edgepatches and a corner patch.

TABLe 4 . We fix the parameter $h=10^{-3}$. The columns below contain the LBB constants on ( $r$-times) refined patches

\begin{tabular}{cccc}
\hline$r$ & Fig. 5 (left) & Fig. 5 (centre) & Fig. 5 (right) \\
\hline 1 & 0.2075 & 0.4693 & 0.3843 \\
2 & 0.2075 & 0.4693 & 0.3205 \\
3 & 0.2075 & 0.4693 & 0.2503 \\
4 & 0.2075 & 0.4693 & 0.1919 \\
\hline
\end{tabular}

\section{Proofs on ReCtangular Meshes}

We start with a preliminary result. This slight generalisation of [Joh16, Theorem 3.89 ] will be the main tool we will base our proof of stability on. 
Lemma 5.1. Let us suppose there exists a space $B \subset L^{2}(\Omega)$, such that

$$
\sup _{\boldsymbol{v} \in \boldsymbol{V}_{\mathcal{P}}} \frac{(q, \operatorname{div} \boldsymbol{v})_{\Omega}}{|\boldsymbol{v}|_{1, \Omega}} \geq \beta_{1}\|q\|_{0, \Omega} \quad \text { for all } \quad q \in B
$$

and a projection $\Pi_{B}: M_{\mathcal{P}} \rightarrow B$ such that

$$
\sup _{\boldsymbol{v} \in \boldsymbol{V}_{\mathcal{P}}} \frac{(q, \operatorname{div} \boldsymbol{v})_{\Omega}}{|\boldsymbol{v}|_{1, \Omega}} \geq \beta_{2}\left\|q-\Pi_{B} q\right\|_{0, \Omega} \quad \text { for all } \quad q \in M_{\mathcal{P}},
$$

where $\beta_{1}, \beta_{2}$ are positive constants. Then $\boldsymbol{V}_{\mathcal{P}} \times M_{\mathcal{P}}$ is inf-sup stable with inf-sup constant $\beta_{0}:=\frac{\beta_{1} \beta_{2}}{1+\beta_{1}+\beta_{2}}$, this is

$$
\sup _{\boldsymbol{v} \in \boldsymbol{V}_{\mathcal{P}}} \frac{(q, \operatorname{div} \boldsymbol{v})_{\Omega}}{|\boldsymbol{v}|_{1, \Omega}} \geq \beta_{0}\|q\|_{0, \Omega} \quad \text { for all } \quad q \in M_{\mathcal{P}} .
$$

Proof. Let $q \in M_{\mathcal{P}}$. Then, using (8) we get

$$
\begin{aligned}
\sup _{\boldsymbol{v} \in \boldsymbol{V}_{\mathcal{P}}} \frac{(q, \operatorname{div} \boldsymbol{v})_{\Omega}}{|\boldsymbol{v}|_{1, \Omega}} & =\sup _{\boldsymbol{v} \in \boldsymbol{V}_{\mathcal{P}}}\left\{\frac{\left(\Pi_{B} q, \operatorname{div} \boldsymbol{v}\right)_{\Omega}}{|\boldsymbol{v}|_{1, \Omega}}+\frac{\left(q-\Pi_{B} q, \operatorname{div} \boldsymbol{v}\right)_{\Omega}}{|\boldsymbol{v}|_{1, \Omega}}\right\} \\
& \geq \beta_{1}\left\|\Pi_{B} q\right\|_{0, \Omega}-\left\|q-\Pi_{B} q\right\|_{0, \Omega} .
\end{aligned}
$$

Multiplying (9) by $\left(\beta_{1}+1\right) / \beta_{2}$ and adding it to the last inequality yields

$$
\left(1+\frac{\beta_{1}+1}{\beta_{2}}\right) \sup _{\boldsymbol{v} \in \boldsymbol{V}_{\mathcal{P}}} \frac{(q, \operatorname{div} \boldsymbol{v})_{\Omega}}{|\boldsymbol{v}|_{1, \Omega}} \geq \beta_{1}\left(\left\|\Pi_{B} q\right\|_{0, \Omega}+\left\|q-\Pi_{B} q\right\|_{0, \Omega}\right) \geq \beta_{1}\|q\|_{0, \Omega},
$$

as required.

5.1. Proof of Theorem 3.1. Our aim is to prove the assumptions of Lemma 5.1. For simplicity of the presentation we will consider a local coordinate system, and will consider $\omega=[-H, H] \times[-h, h+2 H]$ to be partitioned into an edge patch as shown in Fig. 6. We will consider $\omega=M \cup M^{\prime}$, where the "bottom" $M=[-H, H] \times[-h, h]$ is divided into 2 anisotropic elements, as depicted in Fig. 7 (left), or 4 anisotropic elements, as in Fig. 7 (right), respectively. On the other hand, the "top" $M^{\prime}=$ $[-H, H] \times[h, h+2 H]$ is divided into 2 shape-regular elements.
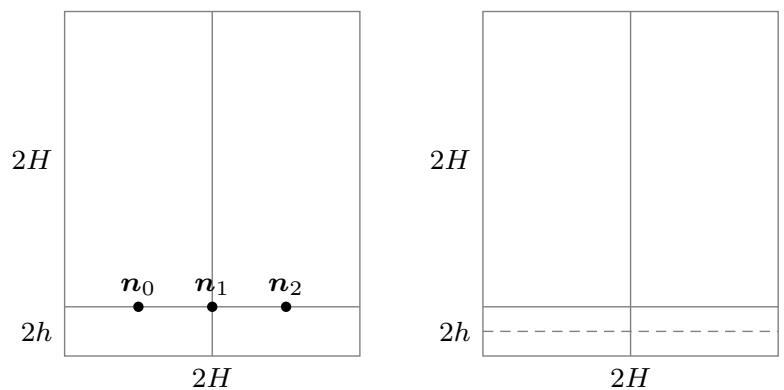

Fig. 6. Edge patches in the local coordinate system. 
For further use we define the following linearly independent functions

$$
\begin{aligned}
& \phi_{0}:=\chi_{M}-\frac{|M|}{\left|M^{\prime}\right|} \chi_{M^{\prime}}, \\
& \phi_{1, M}(x, y):=\frac{x}{H} \chi_{M}(x, y) \text { and } \phi_{2, M}(x, y):=\left(1-\frac{2|x|}{H}\right) \chi_{M}(x, y) .
\end{aligned}
$$

The pressure space $M_{\mathcal{P}}(\omega)$ is included in $\widetilde{M_{\mathcal{P}}}:=M_{\mathcal{P}}\left(M^{\prime}\right) \oplus M_{\mathcal{P}}(M) \oplus \operatorname{span}\left\{\phi_{0}\right\}$. We will prove the inf-sup condition between this larger pressure space and $\boldsymbol{V}_{\mathcal{P}}(\omega)$. As a consequence, the Taylor-Hood pair will be uniformly stable on $\omega$. As a first step, in the next result we state a decomposition of the space $M_{\mathcal{P}}(M)$.
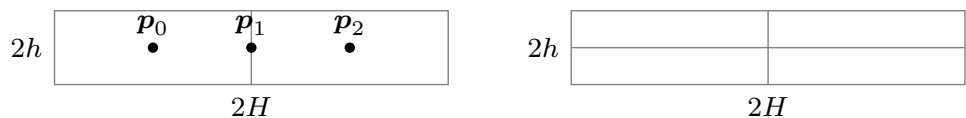

FiG. 7. Flat parts of edge patches.

Lemma 5.2. Let $M=[-H, H] \times[-h, h]$ be partitioned as in Fig. \%. Then, $M_{\mathcal{P}}(M)$ can be decomposed as $M_{\mathcal{P}}(M)=B_{M} \oplus G_{M}$, where

$$
B_{M}:=\left\{q \in M_{\mathcal{P}}(M): \partial_{y} q=0 \text { in } M\right\}=\operatorname{span}\left\{\phi_{1, M}, \phi_{2, M}\right\},
$$

and

$$
G_{M}:=\left\{q \in M_{\mathcal{P}}(M):\left(q, q_{s}\right)_{M}=0 \text { for all } q_{s} \in B_{M}\right\} .
$$

Moreover, for all $v \in H_{0}^{1}(M)$ the following holds

$$
\left(\partial_{y} v, q\right)_{M}=0 \text { for all } q \in B_{M},
$$

and, for every $q \in G_{M}$ there exists $(0, w) \in \boldsymbol{V}_{\mathcal{P}}(M) \cap \boldsymbol{H}_{0}^{1}(M)$ such that

$$
\left(\partial_{y} w, q\right)_{M}=\|q\|_{0, M}^{2} \quad \text { and } \quad|w|_{1, M} \leq C_{1}\|q\|_{0, M},
$$

where $C_{1}$ is independent of the size and shape of the elements in $\mathcal{P}$.

Proof. As it is clear from the context, we will omit the subscript $M$ in this proof. The orthogonality (11) follows using integration by parts. Hence, we only need to prove (12). We split the remainder of the proof in two cases.

Case $1: M=[-H, H] \times[-h, h]$ is split at $x=0$ into two anisotropic cells belonging to $\mathcal{P}$, see Fig. 7 (left). First, we extend the set $\left\{\phi_{1}, \phi_{2}\right\}$ to a basis of $M_{\mathcal{P}}(M)=$ $\operatorname{span}\left\{\phi_{1}, \phi_{2}, \phi_{3}, \phi_{4}, \phi_{5}\right\}$ where $\phi_{1}, \phi_{2}$ are defined as in (10), and

$$
\phi_{3}(x, y):=-\frac{y}{h}, \quad \phi_{4}:=\sqrt{3} \phi_{1} \phi_{3} \quad \text { and } \quad \phi_{5}:=\sqrt{3} \phi_{2} \phi_{3} .
$$

A direct computation, using Fubini's Theorem and the fact that each of the functions $\left\{\phi_{1}, \phi_{2}, \phi_{3}\right\}$ has zero average, yields the orthogonalities

$$
\left(\phi_{i}, \phi_{j}\right)_{M}=\delta_{i j}\left\|\phi_{i}\right\|_{0, M}^{2}=\delta_{i j} \frac{|M|}{3} \quad \text { for } i, j \in\{1, \ldots, 5\},
$$

and then $G_{M}=\operatorname{span}\left\{\phi_{3}, \phi_{4}, \phi_{5}\right\}$, as $\phi_{1}, \phi_{2} \in B_{M}$. To prove (12), let $g_{1} \in G_{M}$, that is, $g_{1}=\sum_{i=3}^{5} q_{i} \phi_{i}$, for some real coefficients $q_{3}, q_{4}, q_{5}$, and $\left\|g_{1}\right\|_{0, M}^{2}=\frac{1}{3}|M| \sum_{i=3}^{5} q_{i}^{2}$. Let $\boldsymbol{p}_{0}, \boldsymbol{p}_{1}, \boldsymbol{p}_{2} \in \stackrel{\circ}{M}$ be the locations of the degrees of freedom (dof) of $\boldsymbol{V}_{\mathcal{P}}(\omega)$ in $\dot{M}$ (see Fig. 7, left), and let $b_{0}, b_{1}, b_{2} \in H_{0}^{1}(M)$ be the piecewise $\mathbb{Q}_{2}$ bubble 
functions, such that $b_{i}\left(\boldsymbol{p}_{j}\right)=\delta_{i j}$ and $\left(0, b_{i}\right) \in \boldsymbol{V}_{\mathcal{P}}(\omega)$. Let, in addition, $v_{3}:=b_{2}+b_{0}$, $v_{4}:=b_{2}-b_{0}$ and $v_{5}:=b_{1}-\frac{1}{4}\left(b_{0}+b_{2}\right)$. A direct calculation using Fubini's theorem gives

$$
\begin{array}{llll}
\left(\partial_{y} v_{3}, \phi_{3}\right)_{M}=\frac{16 H}{9}, & \left(\partial_{y} v_{4}, \phi_{3}\right)_{M}=0, & \left(\partial_{y} v_{5}, \phi_{3}\right)_{M}=0, \\
\left(\partial_{y} v_{3}, \phi_{4}\right)_{M}=0, & \left(\partial_{y} v_{4}, \phi_{4}\right)_{M}=\frac{8 H}{3 \sqrt{3},} & \left(\partial_{y} v_{5}, \phi_{4}\right)_{M}=0, \\
\left(\partial_{y} v_{3}, \phi_{5}\right)_{M}=0, & \left(\partial_{y} v_{4}, \phi_{5}\right)_{M}=0, & \left(\partial_{y} v_{5}, \phi_{5}\right)_{M}=\frac{4 H}{3 \sqrt{3}} .
\end{array}
$$

Hence, defining $v^{\star}=\alpha_{3} q_{3} v_{3}+\alpha_{4} q_{4} v_{4}+\alpha_{5} q_{5} v_{5}$ with $\alpha_{3}=\frac{3 h}{4}, \alpha_{4}=\frac{\sqrt{3} h}{2}$ and $\alpha_{5}=\sqrt{3} h$, we obtain

$$
\left(\partial_{y} v^{\star}, g_{1}\right)_{M}=\sum_{i=3}^{5}\left\|q_{i} \phi_{i}\right\|_{0, M}^{2}=\left\|g_{1}\right\|_{0, M}^{2}
$$

Finally, since $\left|\alpha_{i}\right| \leq \sqrt{3} h$ and $\left|v_{i}\right|_{1, M}^{2} \leq \tilde{C} h^{-2}|M| / 3$, we get

$$
\left|v^{\star}\right|_{1, M}^{2} \leq 3 \sum_{i=3}^{5}\left|\alpha_{i} q_{i} v_{i}\right|_{1, M}^{2} \leq 3 \tilde{C} \sum_{i=3}^{5} q_{i}^{2} h^{2}\left(h^{-2}|M|\right)=9 \tilde{C} \sum_{i=3}^{5}\left\|q_{i} \phi_{i}\right\|_{0, M}^{2},
$$

which, since $\left(0, v^{\star}\right) \in \boldsymbol{V}_{\mathcal{P}}(M)$, finishes the first case with $C_{1}=3 \sqrt{\tilde{C}}$.

Case 2: We now consider the case where $M:=[-H, H] \times[-h, h]$ is divided into four rectangles $K \in \mathcal{P}$ obtained by splitting $M$ along the lines $x=0$ and $y=0$, as depicted in Fig. 7 (right). We extend the set $\left\{\phi_{1}, \phi_{2}, \phi_{3}, \phi_{4}, \phi_{5}\right\}$, introduced in the proof of the first case, to a basis of $M_{\mathcal{P}}(M)$ by adding the following functions

$$
\phi_{6}(x, y):=\left\{\begin{array}{ll}
\phi_{3}(x, 2 y-h) & , \text { if } y>0, \\
-\phi_{3}(x, 2 y+h) & , \text { if } y \leq 0 .
\end{array}, \quad \phi_{7}:=\sqrt{3} \phi_{1} \phi_{6}, \phi_{8}:=\sqrt{3} \phi_{2} \phi_{6} .\right.
$$

These functions are even in $y$, and then, proceeding as before we get

$$
\left(\phi_{i}, \phi_{j}\right)_{M}=\delta_{i j}\left\|\phi_{i}\right\|_{0, M}^{2}=\delta_{i j} \frac{|M|}{3} \quad \text { for } \quad i, j \in\{1, \ldots, 8\} .
$$

Let now $\tilde{g}=g_{1}+g_{2} \in G_{M}$, where $g_{1}=\sum_{i=3}^{5} q_{i} \phi_{i}$, and $g_{2}=\sum_{i=6}^{8} q_{i} \phi_{i}$, for some real coefficients $q_{3}, \ldots, q_{8}$. Thanks to the definition of the function $\phi_{6}$, we observe that $g_{2}$ can be expressed as follows

$$
g_{2}(x, y)= \begin{cases}\left(q_{6} \phi_{3}+q_{7} \phi_{4}+q_{8} \phi_{5}\right)(x, 2 y-h) & , \text { if } y>0 \\ -\left(q_{6} \phi_{3}+q_{7} \phi_{4}+q_{8} \phi_{5}\right)(x, 2 y+h) & \text {, if } y \leq 0 .\end{cases}
$$

Then, using the function $v^{\star}$ from the previous case, we consider the function $\tilde{v}:=$ $v^{\star}+v_{2}^{\star}$, where

$$
v_{2}^{\star}(x, y):= \begin{cases}\left(\alpha_{6} q_{6} v_{3}+\alpha_{7} q_{7} v_{4}+\alpha_{8} q_{8} v_{5}\right)(x, 2 y-h) & , \text { if } y>0, \\ -\left(\alpha_{6} q_{6} v_{3}+\alpha_{7} q_{7} v_{4}+\alpha_{8} q_{8} v_{5}\right)(x, 2 y+h) & , \text { if } y \leq 0,\end{cases}
$$

where $\alpha_{i}, i=6,7,8$ are geometric constants to be determined. We note that $\left(0, v_{2}^{\star}\right) \in \boldsymbol{V}_{\mathcal{P}}(M)$.

Now, we prove $\left(\partial_{y}\left(v^{\star}+v_{2}^{\star}\right), g_{1}+g_{2}\right)_{M}=\|\tilde{g}\|_{0, M}^{2}$. To this end, we recall (14) and since $g_{2}$ and $\partial_{y} v_{2}^{\star}$ are even in $y$, and $g_{1}$ and $\partial_{y} v^{\star}$ are odd in $y$, we obtain the 
orthogonalities

$$
\left(\partial_{y} v_{2}^{\star}, g_{1}\right)_{M}=0 \quad \text { and } \quad\left(\partial_{y} v^{\star}, g_{2}\right)_{M}=0 .
$$

In addition, integrating by substitution and applying (13) gives

$$
\left(\partial_{y} v_{2}^{\star}, g_{2}\right)_{M}=2 \int_{0}^{h} \int_{-H}^{H} \partial_{y} v_{2}^{\star} g_{2} \mathrm{~d} x \mathrm{~d} y=2 \sum_{i=6}^{8} q_{i}^{2} \alpha_{i}\left(\partial_{y} v_{i-3}, \phi_{i-3}\right)_{M},
$$

which on choosing $\alpha_{i}:=\alpha_{i-3} / 2(i=6,7,8)$ gives

$$
\left(\partial_{y}\left(v^{\star}+v_{2}^{\star}\right), \tilde{g}\right)_{M}=\sum_{i=3}^{5}\left\|q_{i} \phi_{i}\right\|_{0, M}^{2}+\sum_{i=6}^{8}\left\|q_{i} \phi_{i-3}\right\|_{0, M}^{2}=\|\tilde{g}\|_{0, M}^{2} .
$$

Finally, we prove $|v|_{1, M} \leq C_{1}\|q\|_{0, M}$. First, applying Cauchy's inequality and considering the scaling (w.r.t. $y$ ) inside $v_{2}^{\star}$ as well as $\alpha_{i}=\alpha_{i-3} / 2$, we get

$$
\left|v_{2}^{\star}\right|_{1, M}^{2} \leq 3\left(4 \sum_{i=6}^{8}\left|\alpha_{i} q_{i} v_{i-3}\right|_{1, M}^{2}\right)=3\left(\sum_{i=6}^{8} q_{i}^{2}\left|\alpha_{i-3} v_{i-3}\right|_{1, M}^{2}\right) \text {. }
$$

Now, using $\left|\alpha_{i} v_{i}\right|_{1, M}^{2} \leq \tilde{C}|M|=3 \tilde{C}\left\|\phi_{i}\right\|_{0, M}^{2}$ (as in Case 1) we get

$$
\left|v_{2}^{\star}\right|_{1, M}^{2} \leq 3 \tilde{C} \sum_{i=6}^{8}\left\|q_{i} \phi_{i-3}\right\|_{0, M}^{2}=9 \tilde{C}\left\|g_{2}\right\|_{0, M}^{2},
$$

where $\tilde{C}$ is the same constant as in (15). Finally, recalling that $v^{\star}$ is even in $y$, and $v_{2}^{\star}$ is odd in $y$, we get $\left(\nabla v^{\star}, \nabla v_{2}^{\star}\right)_{M}=0$ and arrive at

$$
\begin{aligned}
\left|v^{\star}+v_{2}^{\star}\right|_{1, M}^{2} & =\left|v^{\star}\right|_{1, M}^{2}+\left|v_{2}^{\star}\right|_{1, M}^{2} \\
& \leq 9 \tilde{C}\left(\left\|g_{1}\right\|_{0, M}^{2}+\left\|g_{2}\right\|_{0, M}^{2}\right)=9 \tilde{C}\|\tilde{g}\|_{0, M}^{2},
\end{aligned}
$$

which finishes the proof in this case, again with the same constant $C_{1}=3 \sqrt{\tilde{C}}$.

Now we rewrite $\widetilde{M_{\mathcal{P}}}=M_{\mathcal{P}}\left(M^{\prime}\right) \oplus M_{\mathcal{P}}(M) \oplus \operatorname{span}\left\{\phi_{0}\right\}=G_{\omega} \oplus B_{\omega}$ where

$$
G_{\omega}:=M_{\mathcal{P}}\left(M^{\prime}\right) \oplus G_{M} \quad \text { and } \quad B_{\omega}:=B_{M} \oplus \operatorname{span}\left\{\phi_{0}\right\},
$$

with $G_{M}$ and $B_{M}$ defined as in Lemma 5.2. Let

$$
\mathcal{X}:=\boldsymbol{V}_{\mathcal{P}}\left(M^{\prime}\right) \oplus\left\{(0, v) \in \boldsymbol{V}_{\mathcal{P}}(M)\right\} \subset \boldsymbol{V}_{\mathcal{P}}(\omega) .
$$

Then, using Lemma 5.2 and the stability of the Taylor-Hood pair on $M^{\prime}$ (which is due to $M^{\prime}$ being partitioned in a shape regular way), we conclude the uniform inf-sup condition

$$
\inf _{q \in G_{\omega}} \sup _{\boldsymbol{v} \in \mathcal{X}} \frac{(\operatorname{div} \boldsymbol{v}, q)_{\omega}}{\|q\|_{0, \omega}|\boldsymbol{v}|_{1, \omega}} \geq \beta_{2},
$$

where $\beta_{2}$ is independent of the aspect ratio, i.e. the quotient $h / H$. From (19) it follows that $G_{\omega}$ is controlled by a subset of $\boldsymbol{V}_{\mathcal{P}}(\omega)$ that vanishes on $\gamma:=M \cap M^{\prime}$. To control the remaining part of $\widetilde{M_{\mathcal{P}}}$ we need the bubble functions connecting $M$ and $M^{\prime}$. This is stated in the next result.

Lemma 5.3. Let $B_{\omega}$ be defined as in (17). Then, there exists a constant $\beta_{1}>0$ independent of $h$ and $H$, such that

$$
\sup _{\boldsymbol{v} \in \boldsymbol{V}_{\mathcal{P}}(\omega)} \frac{(\operatorname{div} \boldsymbol{v}, q)_{\omega}}{|\boldsymbol{v}|_{1, \omega}} \geq \beta_{1}\|q\|_{0, \omega} \quad \text { for all } \quad q \in B_{\omega} .
$$


Proof. Let $\boldsymbol{n}_{0}, \boldsymbol{n}_{1}, \boldsymbol{n}_{2} \in \gamma$ be the locations of the degrees of freedom (dof) of $\boldsymbol{V}_{\mathcal{P}}(\omega)$ on $\gamma$ (see Fig. 6), and let $f_{0}, f_{1}, f_{2} \in H_{0}^{1}(\omega)$ be the unique piecewise $\mathbb{Q}_{2}$ functions such that $f_{i}\left(\boldsymbol{n}_{j}\right)=\delta_{i j},\left(0, f_{i}\right) \in \boldsymbol{V}_{\mathcal{P}}(\omega)$, and $f_{i}$ are piecewise affine in $y$. Let $q \in B_{\omega}$ be arbitrary, that is, $q=q_{0} \phi_{0}+q_{1} \phi_{1, M}+q_{2} \phi_{2, M}$ for some $q_{0}, q_{1}, q_{2} \in \mathbb{R}$. Let $w^{\star}:=\sum_{i=0}^{2} \alpha_{i} w_{i}$ where $\alpha_{i}$ are coefficients to be chosen and $w_{0}:=f_{0}+f_{2}$, $w_{1}:=f_{2}-f_{0}$ and $w_{2}:=f_{1}-\frac{1}{4}\left(f_{0}+f_{2}\right)$. Using the fact that $\gamma$ consists of two edges of equal lengths, a direct computation gives the orthogonalities

$$
\begin{aligned}
\left(w_{0}, \phi_{1, M}\right)_{\gamma} & =0, & \left(w_{1}, \llbracket \phi_{0} \rrbracket\right)_{\gamma} & =0, & \left(w_{2}, \llbracket \phi_{0} \rrbracket\right)_{\gamma} & =0, \\
\left(w_{0}, \phi_{2, M}\right)_{\gamma} & =0, & \left(w_{1}, \phi_{2, M}\right)_{\gamma} & =0, & \left(w_{2}, \phi_{1, M}\right)_{\gamma} & =0,
\end{aligned}
$$

where $\llbracket \phi_{0} \rrbracket$ is the jump of $\phi_{0}$ across $\gamma$. Then, integration by parts gives

$$
\left(\partial_{y} w^{\star}, q\right)_{\omega}=\left(w^{\star}, \llbracket q \rrbracket\right)_{\gamma}=\alpha_{0} q_{0}\left(w_{0}, \llbracket \phi_{0} \rrbracket\right)_{\gamma}+\sum_{i=1}^{2} \alpha_{i} q_{i}\left(w_{i}, \phi_{i, M}\right)_{\gamma} .
$$

In addition

$$
\begin{aligned}
\left(w_{1}, \phi_{1, M}\right)_{\gamma} & =\frac{2 H}{3}, \quad\left(w_{2}, \phi_{2, M}\right)_{\gamma}=\left(f_{1}, \phi_{2, M}\right)_{\gamma}=\frac{H}{3}, \quad \text { and } \\
\left(w_{0}, \llbracket \phi_{0} \rrbracket\right)_{\gamma} & =\frac{4 H}{3} \llbracket \phi_{0} \rrbracket_{\gamma} .
\end{aligned}
$$

Since $|M| \llbracket \phi_{0} \rrbracket=\left\|\phi_{0}\right\|_{0, \omega}^{2}$ and $\left\|\phi_{i, M}\right\|_{0, \omega}^{2}=\left\|\phi_{i}\right\|_{0, M}^{2}=(2 H / 3) 2 h$, we set $\alpha_{0}:=3 h q_{0}$ $\alpha_{1}=2 h q_{1}$ and $\alpha_{2}=4 h q_{2}$ to obtain

$$
\left(\partial_{y} w^{\star}, q\right)_{\omega}=\|q\|_{0, \omega}^{2} .
$$

Finally, since $\left|\alpha_{i}\right| \leq 4 h q_{i}$ and $\left|w_{i}\right|_{1, \omega}^{2} \leq 3 \sum_{j=0}^{2}\left|f_{j}\right|_{1, \omega}^{2} \leq C\left(h^{-2}|M|+H^{-2}\left|M^{\prime}\right|\right)$, we get

$$
\begin{aligned}
\left|w^{\star}\right|_{1, \omega}^{2} \leq 3 \sum_{i=0}^{2}\left|\alpha_{i} w_{i}\right|_{1, \omega}^{2} \leq C \sum_{i=0}^{2} q_{i}^{2} h^{2}\left(\frac{1}{h^{2}}|M|+\frac{1}{H^{2}}\left|M^{\prime}\right|\right) \\
\quad \leq C \sum_{i=0}^{2} q_{i}^{2}|M|\left(1+\frac{|M|}{\left|M^{\prime}\right|}\right) \leq C \sum_{i=0}^{2}\left\|q_{i} \phi_{i}\right\|_{0, \omega}^{2}=: \beta_{1}^{-2}\|q\|_{0, \omega}^{2},
\end{aligned}
$$

which finishes the proof since $\left(0, w^{\star}\right) \in \boldsymbol{V}_{\mathcal{P}}(\omega)$.

The proof of Theorem 3.1 appears then as an application of Lemma 5.1. In fact, recall the decomposition $M_{\mathcal{P}}(\omega) \subset \widetilde{M_{\mathcal{P}}}=G_{\omega} \oplus B_{\omega}$, where $G_{\omega}, B_{\omega}$ are defined in (17). Let $q \in G_{\omega} \oplus B_{\omega}$, and recall the definition (18) of $\mathcal{X}$. Let also $\Pi_{B} q$ be the $L^{2}(\omega)$ projection of $q$ onto $B_{\omega}$. Then, from (11) and the fact that $\Pi_{B}(q)$ is constant in $M^{\prime}$, we get $\left(\Pi_{B} q, \operatorname{div} \boldsymbol{v}\right)_{\omega}=0$ for all $\boldsymbol{v} \in \mathcal{X}$. Hence

$$
\begin{aligned}
\sup _{\boldsymbol{v} \in \boldsymbol{V}_{\mathcal{P}}} \frac{(q, \operatorname{div} \boldsymbol{v})_{\omega}}{|\boldsymbol{v}|_{1, \omega}} & \geq \sup _{\boldsymbol{v} \in \mathcal{X}} \frac{(q, \operatorname{div} \boldsymbol{v})_{\omega}}{|\boldsymbol{v}|_{1, \omega}} \\
& =\sup _{\boldsymbol{v} \in \mathcal{X}} \frac{\left(q-\Pi_{B} q, \operatorname{div} \boldsymbol{v}\right)_{\omega}}{|\boldsymbol{v}|_{1, \omega}} \geq \beta_{2}\left\|q-\Pi_{B} q\right\|_{0, \omega},
\end{aligned}
$$

where we applied $q-\Pi_{B} q \in G_{\omega}$ and (19). Finally Lemma 5.3 gives

$$
\sup _{\boldsymbol{v} \in \boldsymbol{V}_{\mathcal{P}}} \frac{\left(\Pi_{B} q, \operatorname{div} \boldsymbol{v}\right)_{\omega}}{|\boldsymbol{v}|_{1, \omega}} \geq \beta_{1}\left\|\Pi_{B} q\right\|_{0, \omega}
$$


which finishes the proof upon application of Lemma 5.1 with a constant $\beta=$ $\frac{\beta_{1} \beta_{2}}{1+\beta_{1}+\beta_{2}}$ independent of size and aspect ratios of the edge patch.

Remark 5.1. The essential part of the independence of the constant $\beta$ (in Theorem 3.1) of the aspect ratio is given by the proof of Lemma 5.2. We observed in that proof that, for both cases, the constant $C_{1}$ is given by $3 \sqrt{\tilde{C}}$, and then, it was unaffected by the additional refinement of the edge patch (see the inequalities (15) and (16)). This confirms what was claimed in Remark 3.1, and is affirmed by numerical experiments.

Remark 5.2. It is worth noticing that the proof of Theorem 3.1 provides the inf-sup stability of a family of elements which contains the lowest order Taylor-Hood pair. In fact, the pressure space is allowed to be discontinuous across $\gamma$, which somehow generalises the results known so far.

Remark 5.3. A closer look at the proofs of Lemmas 5.2, 5.3 and Theorem 3.1 shows that the technique can be applied to "overlapped" edge patches, as shown for instance in Fig. 1(f) or Fig. 8 (centre). In this case, Lemma 5.2 has to be applied twice, that is, there are two flat parts, say $M_{1}$ and $M_{2}$, where $M_{2}$ contains the vertically aligned "long and thin" cells. Then, $M_{\mathcal{P}}\left(M_{2}\right)$ has to be decomposed into $B_{M_{2}}=$ $\left\{q \in M_{\mathcal{P}}\left(M_{2}\right): \partial_{x} q=0\right.$ in $\left.M_{2}\right\}$ and $G_{M_{2}}$. Hence, using the same arguments there exists $(v, 0) \in \boldsymbol{V}_{\mathcal{P}}\left(M_{2}\right)$ such that $\left(\partial_{x} v, g\right)_{M_{2}}=\|g\|_{0, M_{2}}^{2}$ and $|v|_{1, M_{2}} \leq C_{1}\|g\|_{0, M_{2}}$ for all $g \in G_{M_{2}}$. Then, similar to (17) and the arguments thereafter, the pair consisting of the velocity space $\mathcal{X}:=\left\{(0, v) \in \boldsymbol{V}_{\mathcal{P}}\left(M_{1}\right)\right\} \oplus \boldsymbol{V}_{\mathcal{P}}\left(M^{\prime}\right) \oplus\left\{(v, 0) \in \boldsymbol{V}_{\mathcal{P}}\left(M_{2}\right)\right\}$ and the pressure space $G_{\omega}:=G_{M_{1}} \oplus M_{\mathcal{P}}\left(M^{\prime}\right) \oplus G_{M_{2}}$ is uniformly inf-sup stable. Then, an adapted proof of Lemma 5.3 shows that $B_{\omega}=B_{M_{1}} \oplus B_{M_{2}} \oplus \operatorname{span}\left\{\phi_{0}, \phi_{0}^{\prime}\right\}$, where $\phi_{0}^{\prime}$ is another piecewise constant function, is controlled by the six bubbles on the edges connecting $M_{1}, M^{\prime}$ and $M_{2}, M^{\prime}$. Finally, the proof of Theorem 3.1 follows analogously considering these modified definitions of $G_{\omega}, B_{\omega}$ and $\mathcal{X}$.

5.2. Proof of Theorem 3.2. In this section we use notations $\omega_{\boldsymbol{c}}, \omega_{E}, \omega$ as suggested in Fig. 8 for the complete and parts of the (refined) corner patch.

As done in the previous section we see that

$$
M_{\mathcal{P}}(\omega) \subset \widetilde{M_{\mathcal{P}}}:=M_{\mathcal{P}}\left(\omega_{E}\right) \oplus M_{\mathcal{P}}\left(\omega_{\boldsymbol{c}}\right) \oplus \operatorname{span}\left\{\phi_{\boldsymbol{c}}\right\}
$$
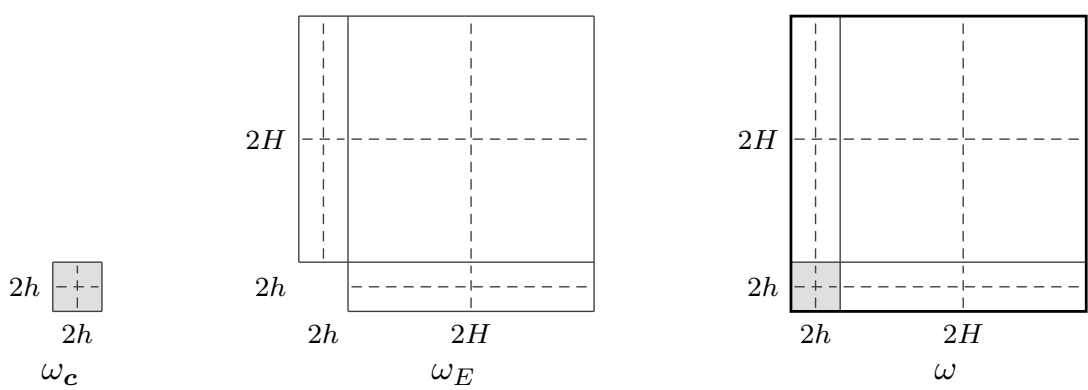

FIG. 8. A corner patch decomposed into $\omega_{c}$ and $\omega_{E}$ satisfying Hypothesis 2 and 1, respectively. 
where $\phi_{\boldsymbol{c}}$ is defined as

$$
\phi_{\boldsymbol{c}}(x, y)= \begin{cases}1 & \text { if }(x, y) \in \omega_{\boldsymbol{c}}, \\ -\frac{\left|\omega_{\boldsymbol{c}}\right|}{\left|\omega_{E}\right|} & \text { otherwise }\end{cases}
$$

We apply the technique developed in [ABW15] to prove Theorem 3.2. Thanks to Theorem 3.1, we know that the pair $\boldsymbol{V}_{\mathcal{P}}\left(\omega_{E}\right) \times M_{\mathcal{P}}\left(\omega_{E}\right)$ is uniformly inf-sup stable, that is, its stability constant does not depend on $H h^{-1}$. In addition, if we recall the Hypothesis 2, then the Taylor-Hood pair is inf-sup stable on $\omega_{c}$.

Let now $q \in M_{\mathcal{P}}(\omega)$, that is, $q=q^{\star}+\Pi_{c} q$, where $q^{\star} \in M_{\mathcal{P}}\left(\omega_{c}\right) \oplus M_{\mathcal{P}}\left(\omega_{E}\right)$ and $\Pi_{c} q \in \operatorname{span}\left\{\phi_{c}\right\}$ is constant on $\omega_{c}$ and $\omega_{E}$. Hence, the inf-sup conditions on $\omega_{c}$ and $\omega_{E}$ (mentioned above) imply

$$
\sup _{\boldsymbol{v} \in \boldsymbol{V}_{\mathcal{P}}(\omega)} \frac{(q, \operatorname{div} \boldsymbol{v})_{\omega}}{|\boldsymbol{v}|_{1, \omega}} \geq \sup _{\boldsymbol{v} \in \boldsymbol{V}_{\mathcal{P}}\left(\omega_{E}\right) \oplus \boldsymbol{V}_{\mathcal{P}}\left(\omega_{\boldsymbol{c}}\right)} \frac{\left(q^{\star}, \operatorname{div} \boldsymbol{v}\right)_{\omega}}{|\boldsymbol{v}|_{1, \omega}} \geq \min \left\{\beta, \beta_{\boldsymbol{c}}\right\}\left\|q^{\star}\right\|_{0, \omega},
$$

where $\beta$ is from Theorem 3.1 and $\beta_{\boldsymbol{c}}$ is from Hypothesis 2 . We will then finish the proof by showing there exists a constant $C>0$ such that

$$
\left\|q^{\star}\right\|_{0, \omega} \geq C\left\|\Pi_{c} q\right\|_{0, \omega} .
$$

To this end, we denote by $\langle q\rangle_{\omega}$ the mean value of $q$ over $\omega \subset \Omega$. Then, we note that the projection $\Pi_{c} q$ is given by

$$
\Pi_{c} q=\langle q\rangle_{\omega_{c}} \chi_{\omega_{c}}+\langle q\rangle_{\omega_{E}} \chi_{\omega_{E}},
$$

and since $0=\langle q\rangle_{\omega}$, we have $\langle q\rangle_{\omega_{E}}=-\left|\omega_{c}\right|\left|\omega_{E}\right|^{-1}\langle q\rangle_{\omega_{c}}$. Hence

$$
\left\|\Pi_{c} q\right\|_{0, \omega}^{2}=\left|\omega_{E}\right|\langle q\rangle_{\omega_{E}}^{2}+\left|\omega_{c}\right|\langle q\rangle_{\omega_{c}}^{2}=\left(\frac{\left|\omega_{c}\right|}{\left|\omega_{E}\right|}+1\right)\left|\omega_{c}\right|\langle q\rangle_{\omega_{c}}^{2}=C_{3}\left|\omega_{c}\right|\langle q\rangle_{\omega_{c}}^{2},
$$

where $C_{3}=|\omega|\left|\omega_{E}\right|^{-1} \geq 1$. Now let $\Gamma_{\boldsymbol{c}}:=\omega_{\boldsymbol{c}} \cap \omega_{E}$, and let $e \subset \Gamma_{\boldsymbol{c}}$ be an arbitrary edge satisfying $e=K_{e} \cap K_{e}^{\prime}$ with $K_{e} \subset \omega_{c}$. Then, from $\langle q\rangle_{\omega_{c}}-\langle q\rangle_{\omega_{E}}=\left\langle\llbracket \Pi_{c} q \rrbracket\right\rangle_{e}$, and since $q$ is continuous in $\omega$, we get

$$
C_{3}\langle q\rangle_{\omega_{c}}=\left\langle\llbracket \Pi_{c} q \rrbracket\right\rangle_{e}=-\left\langle\llbracket q^{\star} \rrbracket\right\rangle_{e} \quad \text { and } \quad\langle q\rangle_{\omega_{c}}^{2}=C_{3}^{-2}\left\langle\llbracket q^{\star} \rrbracket\right\rangle_{e}^{2} .
$$

Then, Cauchy's inequality and [ABW15, Lemma 2.1] yield

$$
\begin{aligned}
\langle q\rangle_{\omega_{c}}^{2} & =\frac{1}{\left|\Gamma_{\boldsymbol{c}}\right|} \int_{\Gamma_{\boldsymbol{c}}}\langle q\rangle_{\omega_{c}}^{2} \\
& =\frac{1}{C_{3}^{2}\left|\Gamma_{\boldsymbol{c}}\right|} \sum_{e \subset \Gamma_{c}} \int_{e}\left\langle\llbracket q^{\star} \rrbracket\right\rangle_{e}^{2} \\
& \leq \frac{1}{C_{3}^{2}\left|\Gamma_{\boldsymbol{c}}\right|} \sum_{e \subset \Gamma_{c}} \int_{e} \frac{1}{|e|}\left\|\llbracket q^{\star} \rrbracket\right\|_{0, e}^{2} \\
& \leq \frac{1}{C_{3}^{2}} \sum_{e \subset \Gamma_{c}} \frac{|e|}{\left|\Gamma_{c}\right|}\left(\frac{1}{\left|K_{e}\right|}+\frac{1}{\left|K_{e}^{\prime}\right|}\right) 4\left\|q^{\star}\right\|_{0, K_{e} \cup K_{e}^{\prime}}^{2} .
\end{aligned}
$$

Now, using $\left|K_{e}\right| \leq\left|K_{e}^{\prime}\right|, \frac{|e|}{\left|\Gamma_{c}\right|} \mid \frac{\left|\omega_{c}\right|}{\left|K_{e}\right|}=1$ and $1 / C_{3} \leq 1$ we get from (20)

$$
\left\|\Pi_{c} q\right\|_{0, \omega}^{2}=C_{3}\left|\omega_{\boldsymbol{c}}\right|\langle q\rangle_{\omega_{\boldsymbol{c}}}^{2} \leq \frac{1}{C_{3}} \sum_{e \subset \Gamma_{\boldsymbol{c}}} \frac{|e|}{\left|\Gamma_{\boldsymbol{c}}\right|} \frac{\left|\omega_{\boldsymbol{c}}\right|}{\left|K_{e}\right|} 8\left\|q^{\star}\right\|_{0, K_{e} \cup K_{e}^{\prime}}^{2} \leq 8\left\|q^{\star}\right\|_{0, \omega}^{2},
$$

as required. 
Remark 5.4. In this proof we have not considered the possibility of refining the corner patches. If we were to consider that case, then in the last step of the above proof we would be led to use the fact that, for a corner patch that has been refined uniformly $r$ times, we have

$$
\frac{|e|}{\left|\Gamma_{\boldsymbol{c}}\right|} \frac{\left|\omega_{\boldsymbol{c}}\right|}{\left|K_{e}\right|}=2^{r-1}
$$

This would give as a result the inf-sup constant

$$
\beta_{\mathcal{P}} \sim C 2^{-\frac{r-1}{2}}
$$

which shows a dependency of the inf-sup constants on the refinement of the partition. This dependency is not an artefact of the proof, as the numerical results in Table 4 show.

\section{The triangulated CASE}

In this section we prove Theorem 3.1 and 3.2 for triangulated edge- and corner patches. A single change is required to prove Theorem 3.2, i.e., we have to replace the used discrete trace estimate by one that is valid on anisotropic triangles, see e.g. [WH03, Theorem 3].

Then it remains to prove Theorem 3.1. To this end, we note that the proofs of Lemma 5.1, Lemma 5.3 remain valid and we only need to prove Lemma 5.2 for triangulated anisotropic (flat) macro elements.

6.1. Proof of Theorem 3.1. We perform a direct computation which is simplified by using the following three-point quadrature formula

$$
\int_{K} f \mathrm{~d} \boldsymbol{x} \approx \frac{|K|}{3} \sum_{i=1}^{3} f\left(\boldsymbol{m}_{i}\right),
$$

where $\boldsymbol{m}_{i}$ are the mid-points of the edges of triangle $K$. This formula is exact for $f \in \mathbb{P}_{2}(K)$.

Now, we let $M=[-H, H] \times[-h, h]$ and notice that however we triangulate $M$, the space $B_{M}=\operatorname{span}\left\{\phi_{1}, \phi_{2}\right\}$ defined in Lemma 5.2 is a subspace of $M_{\mathcal{P}}(M)$. Next we prove (12) on the triangulated macro element.

Case 1: $M$ is split at $x=0$ and then triangulated. Again, we have $\operatorname{dim} G_{M}=3$ and for a basis of $G_{M}$ we choose $\left\{\varphi_{i}\right\}_{i=3}^{5}$ to be the nodal interpolants of $\left\{\phi_{i}\right\}_{i=3}^{5}$ in Lemma 5.2. These functions take the values $+1 /-1 / 0$ at the signs $+/-/ 0$ shown in Fig. 9. For simplicity (but without loss of generality) we chose a triangulation on which $\varphi_{3}, \varphi_{5}$ remain even in $x$, while $\varphi_{4}$ is odd in $x$. Additionally the product $\varphi_{3} \varphi_{5}$ vanishes in each of the quadrature points, and therefore $\left\{\varphi_{i}\right\}_{i}$ is an orthogonal basis of $G_{M}$. Hence, for $g \in G_{M}$ with $g=\sum_{i=3}^{5} q_{i} \varphi_{i}$, the quadrature formula yields

$$
\|g\|_{0, M}^{2}=\sum_{i=3}^{5}\left\|q_{i} \varphi_{i}\right\|_{0, M}^{2}=\frac{|M|}{6}\left(2 q_{3}^{2}+q_{4}^{2}+2 q_{5}^{2}\right) .
$$

Now, let $b_{0}, b_{1}, b_{2}$ be the quadratic bubble functions, each supported on two triangles and taking the value 1 at the midpoint of the shared edge. Then defining $v_{3}, v_{4}, v_{5}$ similar to Lemma 5.2 by $v_{3}:=b_{2}+b_{0}, v_{4}:=b_{2}-b_{0}$ and $v_{5}:=2 b_{1}-\left(b_{2}+b_{0}\right)$ we get

$$
\left(\partial_{y} v_{i}, g\right)_{M}=-\left(v_{i}, \partial_{y} g\right)_{M}=-q_{i}\left(v_{i}, \partial_{y} \varphi_{i}\right)_{M} \quad \text { for } \quad i=3,4,5,
$$




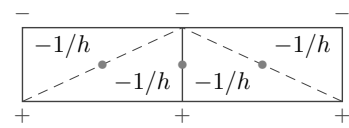

$\varphi_{3}$

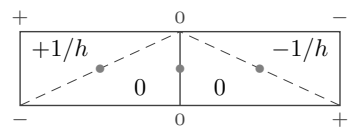

$\varphi_{4}$

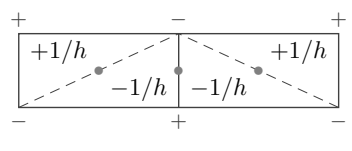

$\varphi_{5}$

FIG. 9. Basis of $G_{M}$ with sign-patterns of $\varphi_{i}$ and $\partial_{y} \varphi_{i}$.

and then $v^{\star}:=\sum_{i=3}^{5} \alpha_{i} v_{i}$ satisfies

$$
\left(\partial_{y} v^{\star}, g\right)_{M}=-\left(v^{\star}, \partial_{y} g\right)_{M}=-\sum_{i=3}^{5} \alpha_{i} q_{i}\left(v_{i}, \partial_{y} \varphi_{i}\right)_{M} .
$$

To calculate these products we use the quadrature formula and the values of $\left.\left(\partial_{y} \varphi_{i}\right)\right|_{K}$, given inside the triangles shown in Fig. 9. We obtain

$$
\begin{aligned}
& \left(v_{3}, \partial_{y} \varphi_{3}\right)_{M}=-|M| /(3 h), \\
& \left(v_{4}, \partial_{y} \varphi_{4}\right)_{M}=-|M| /(6 h), \\
& \left(v_{5}, \partial_{y} \varphi_{5}\right)_{M}=\left(2 b_{1}, \partial_{y} \varphi_{5}\right)_{M}=-|M| /(3 h),
\end{aligned}
$$

and choosing $\alpha_{i}=-q_{i} h$ we obtain

$$
\left(\partial_{y} v^{\star}, g\right)_{M}=\sum_{i=3}^{5}\left\|q_{i} \varphi_{i}\right\|_{0, M}^{2}=\|g\|_{0, M}^{2} .
$$

Finally, since $\left|\alpha_{i}\right| \leq h q_{i}$ and $\left|v_{i}\right|_{1, M}^{2} \leq C h^{-2}|M|$ we get

$$
\left|v^{\star}\right|_{1, M}^{2} \leq C \sum_{i=3}^{5}\left|\alpha_{i} v_{i}\right|_{1, M}^{2} \leq C \sum_{i=3}^{5} h^{2} q_{i}^{2}\left(h^{-2}|M|\right)=C\|g\|_{0, M}^{2},
$$

which finishes case 1 as $\left(0, v^{\star}\right) \in \boldsymbol{V}_{\mathcal{P}}(M)$.

Case 2: On appropriate triangulations of a 2-by-2 macro element one can construct orthogonal bases of the velocity and pressure spaces, again even and odd on $y$. Then, the proof follows a similar way to Case 1 .

6.2. A numerical confirmation. The results presented in Table 5 show that the Taylor-Hood pair is also uniformly stable on triangulated edge and corner patches. Furthermore, we see that the difference between the inf-sup constants in column 1 and 2 is marginal, that is, the additional refinement does not affect the inf-sup constant. On the other hand, we show that hypothesis 1 for edge patches must be satisfied for their triangulated versions, as Fig. 11 confirms.
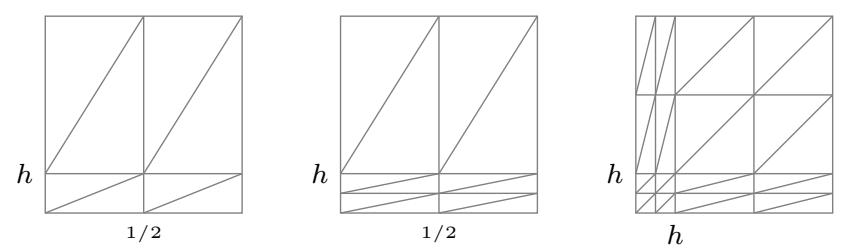

FIG. 10. Triangulated edge and corner patches satisfying the hypotheses of Theorems 3.1 and 3.2. In all cases, $\Omega=(0,1)^{2}$. 
TABle 5. Discrete inf-sup constants of the $\mathbb{P}_{2}^{2} \times \mathbb{P}_{1}$ pair on the partitions shown in Fig. 10

\begin{tabular}{cccc}
\hline$h$ & Fig. 10(left) & Fig. 10(centre) & Fig. 10(right) \\
\hline $10^{-1}$ & 0.3346 & 0.3332 & 0.3844 \\
$10^{-2}$ & 0.2690 & 0.2677 & 0.3744 \\
$10^{-3}$ & 0.2480 & 0.2478 & 0.3519 \\
$10^{-4}$ & 0.2453 & 0.2452 & 0.3493 \\
$10^{-5}$ & 0.2450 & 0.2450 & 0.3491 \\
\hline
\end{tabular}

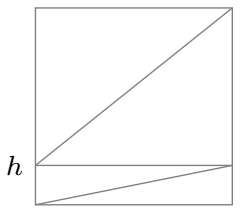

\begin{tabular}{cc}
\hline$h$ & $\beta_{\mathcal{P}}$ \\
\hline $10^{-1}$ & $1.788 \cdot 10^{-1}$ \\
$10^{-2}$ & $8.236 \cdot 10^{-2}$ \\
$10^{-3}$ & $2.725 \cdot 10^{-2}$ \\
$10^{-4}$ & $8.656 \cdot 10^{-3}$ \\
$10^{-5}$ & $2.739 \cdot 10^{-3}$ \\
\hline
\end{tabular}

Fig. 11. A triangulated edge patch that does not satisfy Hypothesis 1 and the associated discrete inf-sup constants for the $\mathbb{P}_{2} \times \mathbb{P}_{1}$ pair.

\section{Possible extensions}

In this work we have proven the uniform inf-sup stability of the lowest order Taylor-Hood pair in a family of anisotropic meshes. Up to our best knowledge, this is the first proof available for this pair on anisotropic meshes. The numerical evidence shown suggests that the hypotheses made for the partitions (macro elements) are minimal, so the results presented here are optimal.

There are, nevertheless, open questions. The first is the possible extension to higher order polynomials. Another possible extension is the possibility to allow geometric refinements towards a corner of a given partition. The proof of Theorem 3.2 would not fail in this situation provided the result on geometric edge patches holds. In fact, a dependency on a level of refinement as documented in Remark 5.4 would not occur. Another important open question is the validity of our result in the case the meshes do not have an underlying structure, and the case of non-affine quadrilaterals. Finally, the problem of stability of an anisotropic refinement strategy, driven by a posteriori error estimators, is also a topic of interest. All these constitute open questions that will be subject of future research.

\section{REFERENCES}

[ABW15] M. Ainsworth, G. R. Barrenechea, and A. Wachtel, Stabilization of high aspect ratio mixed finite elements for incompressible flow, SIAM J. Numer. Anal. 53 (2015), no. 2, $1107-1120$.

[AC00] M. Ainsworth and P. Coggins, The stability of mixed hp-finite element methods for Stokes flow on high aspect ratio elements, SIAM J. Numer. Anal. 38 (2000), no. 5, 1721-1761 (electronic). 
[AD99] G. Acosta and R. G. Durán, The maximum angle condition for mixed and nonconforming elements: application to the Stokes equations, SIAM J. Numer. Anal. 37 (1999), no. $1,18-36$ (electronic).

[AMR03] T. Apel and H. Maharavo Randrianarivony, Stability of discretizations of the Stokes problem on anisotropic meshes, Math. Comput. Simulation 61 (2003), no. 3-6, 437-447.

[ANS01] T. Apel, S. Nicaise, and J. Schöberl, Crouzeix-Raviart type finite elements on anisotropic meshes, Numer. Math. 89 (2001), no. 2, 193-223.

[BBF13] D. Boffi, F. Brezzi, and M. Fortin, Mixed finite element methods and applications, Springer Series in Computational Mathematics, vol. 44, Springer, Heidelberg, 2013.

[BF91] F. Brezzi and R. S. Falk, Stability of higher-order Hood-Taylor methods, SIAM J. Numer. Anal. 28 (1991), no. 3, 581-590.

[BLR12] M. Braack, G. Lube, and L. Röhe, Divergence preserving interpolation on anisotropic quadrilateral meshes, Comput. Methods Appl. Math. 12 (2012), no. 2, 123-138.

[Bof94] D. Boffi, Stability of higher order triangular Hood-Taylor methods for the stationary Stokes equations, Math. Models Methods Appl. Sci. 4 (1994), no. 2, 223-235.

[Bof97] D. Boffi, Three-dimensional finite element methods for the Stokes problem, SIAM J. Numer. Anal. 34 (1997), no. 2, 664-670.

[BP79] M. Bercovier and O. Pironneau, Error estimates for finite element method solution of the stokes problem in the primitive variables, Numer. Math. 33 (1979), no. 2, 211-224.

[Che14] L. Chen, A simple construction of a Fortin operator for the two dimensional TaylorHood element, Comput. Math. Appl. 68 (2014), no. 10, 1368 - 1373.

[DL08] R. G. Durán and A. L. Lombardi, Error estimates for the Raviart-Thomas interpolation under the maximum angle condition, SIAM J. Numer. Anal. 46 (2008), no. 3, 14421453.

[Dur08] R. G. Durán, Mixed finite element methods, Mixed finite elements, compatibility conditions, and applications, Lectures given at the C.I.M.E. Summer School held in Cetraro, June 26-July 1, 2006 (Daniele Boffi and Lucia Gastaldi, eds.), Springer-Verlag, Berlin; Fondazione C.I.M.E., Florence, 2008, pp. 1-44.

[Fal08] R. Falk, A Fortin operator for two-dimensional Taylor-Hood elements, M2AN Math. Model. Numer. Anal. 42 (2008), no. 3, 411-424.

[GR86] V. Girault and P.-A. Raviart, Finite element methods for Navier Stokes equations, Springer, Berlin, 1986.

[Joh16] V. John, Finite element methods for incompressible flow problems, Springer, Berlin, 2016.

[MSW13] K. Mardal, J. Schöberl, and R. Winther, A uniformly stable Fortin operator for the Taylor-Hood element, Numer. Math. 123 (2013), no. 3, 537-551.

[SS98] D. Schötzau and C. Schwab, Mixed hp-FEM on anisotropic meshes, Math. Models Methods Appl. Sci. 8 (1998), no. 5, 787-820.

[SSS99] D. Schötzau, C. Schwab, and R. Stenberg, Mixed hp-FEM on anisotropic meshes. II. Hanging nodes and tensor products of boundary layer meshes, Numer. Math. 83 (1999), no. 4, 667-697.

[Ste90] R. Stenberg, Error analysis of some finite element methods for the Stokes problem, Mathematics of Computation 54 (1990), no. 190, 495-508.

[TH73] C. Taylor and P. Hood, A numerical solution of the Navier-Stokes equations using the finite element technique, Computers \& Fluids 1 (1973), no. 1, 73 - 100.

[Ver84] R. Verfürth, Error estimates for a mixed finite element approximation of the Stokes equations, RAIRO Anal. Numér. 18 (1984), no. 2, 175-182.

[WH03] T. Warburton and J. S. Hesthaven, On the constants in hp-finite element trace inverse inequalities, Comput. Methods Appl. Mech. Engrg. 192 (2003), no. 25, 2765-2773.

Department of Mathematics and Statistics, University of Strathclyde, 26 Richmond Street, Glasgow G1 1XH, Scotland

E-mail address: gabriel.barrenechea@strath.ac.uk

(corresponding author) Department of Mathematics, ITAM, Río Hondo 1, Ciudad de MÉxico 01080, MExico

E-mail address: andreas.wachtel@itam.mx 